\title{
Michelson interferometer composite cavity fiber laser sensor with radio frequency interrogation
}

\author{
Xu Wang (王 旭), Enlai Wang (王恩来) ${ }^{*}$, and Fang Wang (王 芳) \\ College of Electronic and Electrical Engineering, Henan Normal University, Xinxiang 453007, China \\ *Corresponding author: wangenlai1996@126.com \\ Received December 22, 2021 | Accepted January 21, 2022 | Posted Online February 16, 2022
}

\begin{abstract}
A Michelson interferometer (MI) composite cavity fiber laser sensing system based on radio frequency (RF) interrogation is proposed and experimentally demonstrated. The system down-converts the traditional MI light frequency detection to RF detection, which improves the stability of the system. The optic fiber MI is placed in the laser resonator to form a composite cavity structure, which greatly improves the sensitivity of beat frequency signal demodulation.
\end{abstract}

Keywords: Michelson interferometer; compound cavity; multi-longitudinal mode beat frequency; radio-frequency detection. DOI: 10.3788/COL202220.040603

\section{Introduction}

Fiber optic interferometers based on the Michelson structure have been widely studied and applied to temperature ${ }^{[1]}$, stress $^{[2]}$, liquid level ${ }^{[3]}$, vibration ${ }^{[4]}$, and other sensing. Compared with conventional sensors, interference-type sensors have the advantages of high sensitivity and simple structure ${ }^{[5]}$. The traditional method of demodulating the MI system is to obtain sensing information by detecting the phase change of light ${ }^{[6-8]}$ and then converting the phase change into light intensity change ${ }^{[9]}$, which is generally carried out in the optical domain. Although this method has high sensitivity, it has poor immunity from interference ability.

In 1996, Kringlebotn et al. proposed using the laser beat technique to interrogate the wavelength variation of two orthogonal polarization modes in the laser ${ }^{[10]}$. The fiber laser beat frequency interrogation technology is commonly used to convert the wavelength shift into an electrical signal for easy reading and realtime monitoring due to its cheap and stable characteristics. The distributed feedback (DFB) fiber lasers and distributed Bragg reflector (DBR) fiber lasers are used to detect the sensing parameters by measuring the beat frequency signal (BFS), which is generated by two polarization modes ${ }^{[11,12]}$. However, it is difficult to control the polarization of fiber lasers. Thus, the appropriate BFS between the two polarization modes is not easy to obtain. In recent years, the multi-longitudinal mode (MLM) fiber laser sensing system based on laser beat frequency demodulation is proposed due to MLMs being easier to establish in a laser cavity. By monitoring the BFS between two different longitudinal modes, measurement of temperature, stress, etc. is achieved. In 2010, Liu et al. constructed an MLM fiber laser sensor with a resonant laser cavity consisting of two fiber gratings and an erbium-doped fiber (EDF) and tested it for stress sensing ${ }^{[13]}$. The MLM BFS demodulation technology can reduce the influence of fiber laser mode hopping. When one longitudinal mode jumps, the other modes can still generate the BFS in two phases, so the BFS is relatively stable. This demodulation method realizes sensing by detecting the BFS, which is carried out in the electrical domain and has high stability ${ }^{[14-17]}$.

In this paper, we propose a Michelson interferometer (MI) composite cavity fiber laser sensing system with radio-frequency (RF) detection. Unlike other composite cavity sensing systems ${ }^{[18,19]}$, the MI is used as a cavity mirror of the laser cavity, and the fiber Bragg grating (FBG) is used as another cavity mirror. The system converts the change of MI arm length difference into the shift of interference wavelength, which is demodulated by tracking the shift of the BFS envelope. This system downconverts the light frequency detection to RF detection, simplifies the experimental structure, and improves the stability of the system. At the same time, the MI is used to form a composite cavity structure to improve the sensitivity of optical fiber sensing.

\section{Experimental Structure and Principle}

Figure 1 illustrates the schematic diagram of the compound laser system. The composite cavity fiber laser consists of a linear cavity, which is made of a piece of EDF pumped by a $980 \mathrm{~nm}$ pump source through a wavelength-division multiplexer (WDM) to provide stable enough gain for multiple mode lasing, and two reflectors. One of the reflectors is an FBG that can be used as a filter of the system. Another reflector is an MI, which includes an optical coupler (OC), two pieces of single mode fiber (SMF), and two Faraday rotating mirrors (FRMs) to form a composite cavity structure. Using the FRM as a reflector can control the 


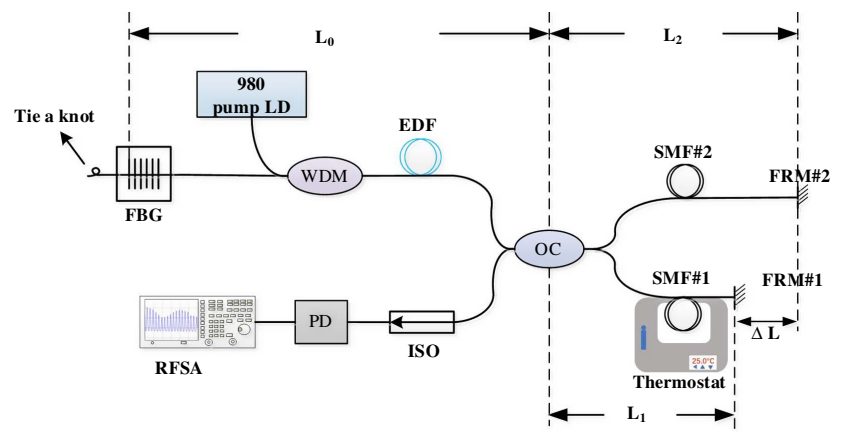

Fig. 1. Schematic diagram of the system. FBG, fiber Bragg grating; WDM, wavelength division multiplexer; EDF, erbium-doped fiber; OC, optical coupler; SMF, single-mode fiber; FRM, Faraday rotating mirror; ISO, fiber optical isolator; $\mathrm{PD}$, photo-detector; RFSA, radio-frequency spectrum analyzer.

polarization states of the two reflected beams so as to obtain a better interference effect. SMF\#1 and SMF\#2 are used as the sensing arm and the reference arm, respectively, and the length difference is $\Delta L$.

The light entering the resonant cavity is split into two paths through the 50/50 OC. One beam propagating along SMF\#1 is reflected by FRM\#1; the other beam propagating along SMF\#2 is reflected by FRM\#2. Then, the two reflected lights interfere in the OC, and the output light spectrum after the interference is related to the optical path difference. The interference spectrum and the longitudinal mode of the fiber laser are superimposed on the PD to form a laser BFS with envelope modulation. The MI converts the variation of arm length difference into the change of interference wavelength. The BFS envelope varies with the interference wavelength changing.

The cavity mode of the laser cavity is shown in Fig. 2(a), and the longitudinal mode in the cavity is expressed as ${ }^{[13]}$

$$
V_{m}=m \cdot \frac{c}{2 n_{\mathrm{eff}} L}, \quad m=1,2, \ldots
$$

where $L=L_{0}+L_{1}$ is the cavity length of the composite cavity fiber laser, which consists of two segments, one of which is $L_{0}$ from the gate area of the FBG to the OC, and the other is the short arm length $L_{1}$ of the MI, $n_{\text {eff }}$ is the effective refractive index of the fiber, $c$ is the light velocity in vacuum, and $m$ is the laser mode number.

As shown in Fig. 2(b), the interference spectrum of the MI causes the envelope modulation of longitudinal mode frequency. By the selection of the interference spectrum of the MI, only those longitudinal modes that exceed the threshold value can generate laser output, and the selected longitudinal modes are shown in Fig. 2(c).

The BFS envelope generated by the composite cavity is obtained from the mutual beat between the longitudinal modes that exceed the threshold in Fig. 2(c), as shown in Fig. 3.

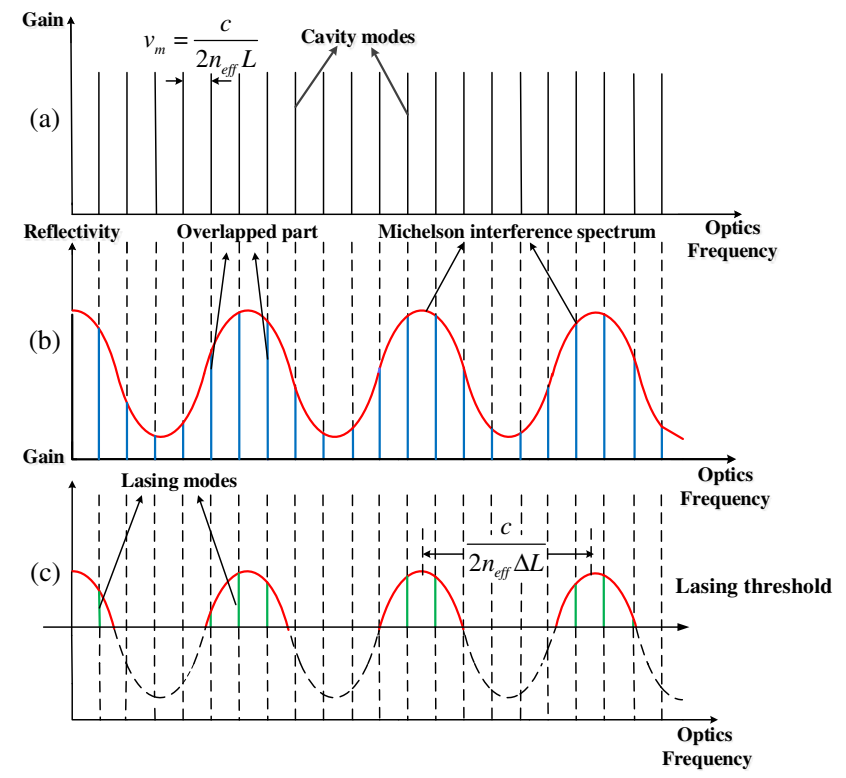

Fig. 2. Schematic view of the laser modes: (a) the cavity modes of the laser cavity; (b) interference spectrum of the MI and overlap with the laser cavity modes; (c) modes of the composite cavity.

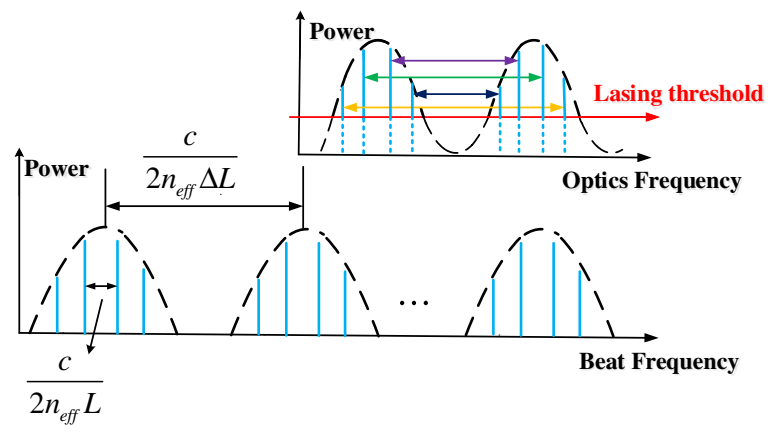

Fig. 3. BFS envelope generated by the composite cavity.

The BFS envelope peak is expressed as

$$
V_{q}=q \cdot \frac{c}{2 n_{\mathrm{eff}} \Delta L}, \quad q=1,2, \ldots
$$

When the MI arm length difference $\Delta L$ changes, the change of BFS envelope interval $\Delta f$ can be described as the following formula:

$$
\begin{gathered}
\frac{\Delta f}{f}=\frac{\delta\left(n_{\mathrm{eff}} \Delta L\right)}{n_{\mathrm{eff}} \Delta L}, \\
\Delta f=f \cdot \frac{\delta\left(n_{\mathrm{eff}} \Delta L\right)}{n_{\mathrm{eff}} L} \cdot \frac{L}{\Delta L} .
\end{gathered}
$$

In Eq. (4), $\delta\left(n_{\text {eff }} \Delta L\right) / n_{\text {eff }} L$ is the relative change in the length of the laser cavity, and $\Delta f$ is the BFS envelope interval. Compared with literature ${ }^{[20]}$, there is more $L / \Delta L$ in the 
equation because the system is a composite cavity structure. The MI arm length difference changes, and, correspondingly, the system resonant cavity will also change. In this way, the sensitivity is increased by $L / \Delta L$ times. At the same time, it can be seen in the following comparative experiments that under the same conditions reducing the arm length difference of the MI will correspondingly improve the sensitivity of the system.

\section{Experimental Results and Analysis}

The structure of this system is shown in Fig. 1. In the experiments, the FBG has the reflectivity of $90 \%$, Bragg wavelength of $1549.83 \mathrm{~nm}$, and $3 \mathrm{~dB}$ bandwidth of $0.077 \mathrm{~nm}$. The end of the FBG is knotted to reduce the influence of external light on the system. The total cavity length of the laser resonator is $86 \mathrm{~m}$, and the $\mathrm{EDF}$ is $6 \mathrm{~m}$. The $980 \mathrm{~nm}$ pump source is launched into the resonant cavity through the WDM. In addition, an optical isolator is connected after the output port of the $\mathrm{OC}$ to ensure one-way transmission of the laser. The BFS, which is generated by the MLM, is detected by a photo-detector (PD) and observed by an RF spectrum analyzer.

As a comparison, the arm length difference $\Delta L$ of the MI was set to $0.38 \mathrm{~m}$ and $0.28 \mathrm{~m}$, respectively. The BFS envelope spectrum of this system in room condition is shown in Fig. 4, in the spectral width range of $0-1.5 \mathrm{GHz}$. Among them, Fig. 4(a) shows the BFS envelope spectrum when the arm length difference is $0.38 \mathrm{~m}$, and the BFS envelope interval $\Delta v_{q}$ is about $272 \mathrm{MHz}$. According to Eq. (2), the $\Delta L$ is $0.38 \mathrm{~m}$, which is in good agreement with the set arm length difference $\Delta L$. Figure 4(b) shows the BFS envelope spectrum when the arm length difference is $0.28 \mathrm{~m}$, and the BFS envelope interval $\Delta v_{q}$ is about $367.5 \mathrm{MHz}$. According to Eq. (2), the $\Delta L$ is $0.28 \mathrm{~m}$, which also coincides with the set arm length difference $\Delta L$.

The BFS envelope with the frequency of $1092 \mathrm{MHz}$ in Fig. 4(a) and the BFS envelope with the frequency of $1106 \mathrm{MHz}$ in Fig. 4(b) are chosen as the observation objects. When the spectral width is set to $60 \mathrm{MHz}$, the composition of the BFS envelope details corresponding to each is shown in Fig. 5.

As can be seen from Figs. 5(a) and 5(b), the longitudinal mode spacings of the BFS envelope details are both 1.2 MHz. The difference is that Fig. 5(b) has a higher number of longitudinal

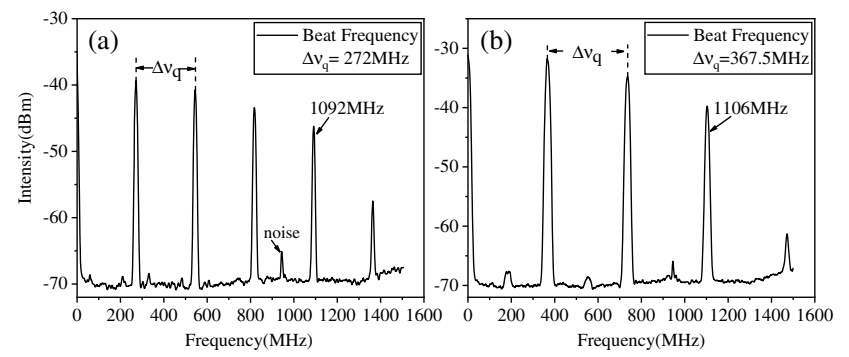

Fig. 4. BFS envelope spectrum under different arm length differences: (a) the BFS envelope when the $\Delta L$ is $0.38 \mathrm{~m}$; (b) the BFS envelope when the $\Delta L$ is $0.28 \mathrm{~m}$.

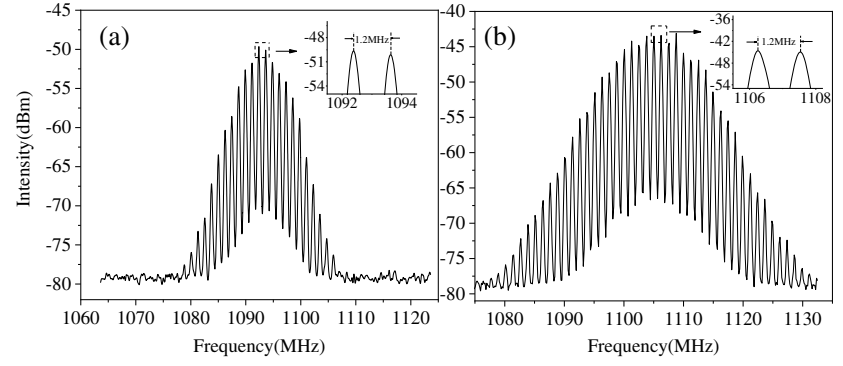

Fig. 5. Details of the selected observation BFS envelope: (a) the BFS envelope with center frequency of $1092 \mathrm{MHz}$; (b) the BFS envelope with center frequency of $1106 \mathrm{MHz}$.

modes compared to Fig. 5(a). This is because the laser cavity length of the system is unchanged, and only the arm length difference of MI is changed, so the shorter the arm length difference, the larger the number of longitudinal modes. In addition, according to Eq. (1), the longitudinal mode spacing corresponding resonator cavity length $L$ is $86.2 \mathrm{~m}$, which is basically consistent with the set system cavity length of $86 \mathrm{~m}$.

As a sensing arm, SMF\#1 was fixed in a thermostat, and the sensing arm was the same for both sets of experiments, as shown in Fig. 1. Except for the part exposed to the outside of the instrument, about $69 \mathrm{~m}$ was heated in the experiment. The inner temperature of the thermostat can be detected by a thermo-couple with an accuracy of $\pm 0.1^{\circ} \mathrm{C}$. The thermal expansion of the fiber leads to the lengthening of $L_{1}$, resulting in a decrease in $\Delta L$. According to Eq. (4), the decrease in $\Delta L$ results in a large $\Delta f$, leading to the MLM resonance at a higher frequency. In other words, as the temperature increases, the BFS envelope can be observed to move to the right on the RF spectrum analyzer.

The shifts of the selected BFS envelope with temperature are shown in Fig. 6. In particular, Fig. 6(a) displays the shift of the BFS envelope detail near $1092 \mathrm{MHz}$ as the temperature increases with $25^{\circ} \mathrm{C}$ intervals. It can be seen that the BFS envelope increased from $1092.00 \mathrm{MHz}$ to $1305.85 \mathrm{MHz}$ as the temperature increased from $25^{\circ} \mathrm{C}$ to $150^{\circ} \mathrm{C}$. Since there are a large number of longitudinal modes in the BFS envelope near $1106 \mathrm{MHz}$, which are not easy to observe, the BFS envelope with a spectral width of $300 \mathrm{MHz}$ is selected for observation, as shown in Fig. 6(b). The figure records the shift of the BFS envelope as
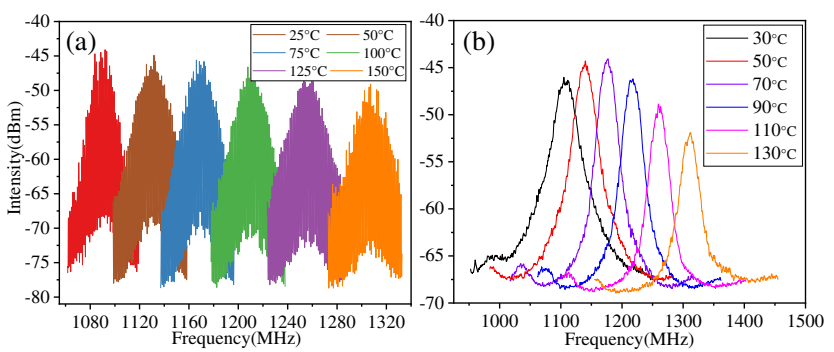

Fig. 6. Shift of BFS envelope with increasing temperature: (a) the shift of the BFS envelope with center frequency of $1092 \mathrm{MHz}$ (b) the shift of the BFS envelope with center frequency of $1106 \mathrm{MHz}$. 
the temperature increases with $20^{\circ} \mathrm{C}$ intervals. It can be seen that the BFS envelope increased from $1106.00 \mathrm{MHz}$ to $1310.25 \mathrm{MHz}$ as the temperature increased from $30^{\circ} \mathrm{C}$ to $130^{\circ} \mathrm{C}$.

The responses of the measured BFS envelope to the temperature are shown in Fig. 7. In particular, Fig. 7(a) shows that the frequency varies linearly with temperature from $25^{\circ} \mathrm{C}$ to $150^{\circ} \mathrm{C}$. The slope of the linear fitting is $1.68 \mathrm{MHz} /{ }^{\circ} \mathrm{C}$ at $1092 \mathrm{MHz}$, and the fitting degree is $99.491 \%$. Figure $7(\mathrm{~b})$ shows that the frequency varies linearly with temperature from $30^{\circ} \mathrm{C}$ to $130^{\circ} \mathrm{C}$. The slope of the linear fitting is $2.04 \mathrm{MHz} /{ }^{\circ} \mathrm{C}$ at $1106 \mathrm{MHz}$, and the fitting degree is $99.369 \%$.

In the experiment of the fiber ring laser sensor for temperature measurement by Yin et al. ${ }^{[21]}$, the sensitivity of the BFS is $3.02 \mathrm{kHz} /{ }^{\circ} \mathrm{C}$ at $525.8 \mathrm{MHz}$ and $8.48 \mathrm{kHz} /{ }^{\circ} \mathrm{C}$ at $1480.5 \mathrm{MHz}$ after heating about $16 \mathrm{~m}$ of SMF. The literature ${ }^{[9]}$ proposed a composite cavity structure that improves the sensitivity through the Vernier effect, heating about $18 \mathrm{~m}$ of SMF, and the sensitivity of the BFS envelope is $176.67 \mathrm{kHz} /{ }^{\circ} \mathrm{C}$ at $436 \mathrm{MHz}$ and $299.17 \mathrm{kHz} /{ }^{\circ} \mathrm{C}$ at $510 \mathrm{MHz}$. In our experiment, the MI structure is used to form a composite cavity system. When the $69 \mathrm{~m} \mathrm{SMF}$ is heated, the sensitivity can reach $2.04 \mathrm{MHz} /{ }^{\circ} \mathrm{C}$ at $1106 \mathrm{MHz}$. As a comparison, the sensitivity of this work has been greatly improved.

In the experiment, the BFS envelope sensor signals are stable, although they may be disturbed by the environmental perturbations. Figure 8 shows a change in the BFS envelope of $1142 \mathrm{MHz}$ over time. The BFS envelope is measured every $10 \mathrm{~min}$ for $50 \mathrm{~min}$ at $50^{\circ} \mathrm{C}$. The center frequency of the BFS envelope is basically stable and hardly changes.

Figure 9(a) shows that the sensor fiber is tested for temperature drop, and the temperature is gradually decreased from $130^{\circ} \mathrm{C}$ to $30^{\circ} \mathrm{C}$. The BFS envelope near $1106 \mathrm{MHz}$ is recorded every $20^{\circ} \mathrm{C}$. It can be seen that the BFS decreases from $1309.50 \mathrm{MHz}$ to $1106.25 \mathrm{MHz}$ as the temperature decreases $100^{\circ} \mathrm{C}$. Figure 9 (b) shows the comparison of fitting curves of temperature rises and drops, and their fitting degrees are $99.369 \%$ and $99.525 \%$, respectively. Their sensitivities are $2.04 \mathrm{MHz} /{ }^{\circ} \mathrm{C}$ at $1106 \mathrm{MHz}$ and $2.03 \mathrm{MHz} /{ }^{\circ} \mathrm{C}$ at $1106 \mathrm{MHz}$, respectively. The two fitting curves are basically identical, which further shows that the system has good stability and high sensitivity.
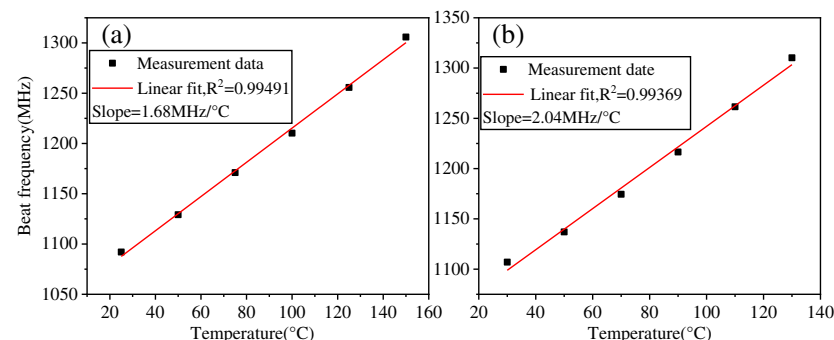

Fig. 7. Fitting result of frequency corresponding to the temperature change: (a) fitting result of the BFS envelope with center frequency of $1092 \mathrm{MHz}$; (b) fitting result of the BFS envelope with center frequency of $1106 \mathrm{MHz}$.

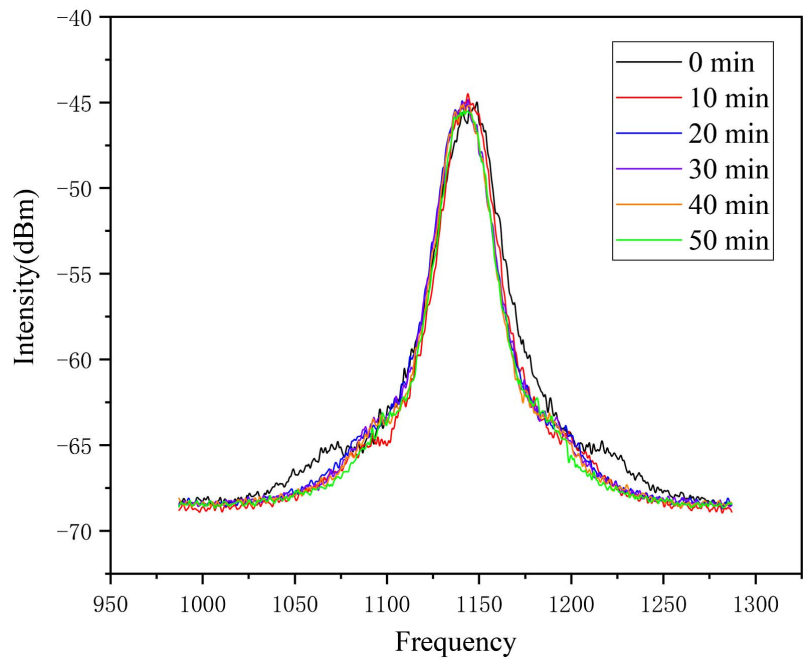

Fig. 8. Stability of the BFS envelope under $50^{\circ} \mathrm{C}$ measured every $10 \mathrm{~min}$ for $50 \mathrm{~min}$.
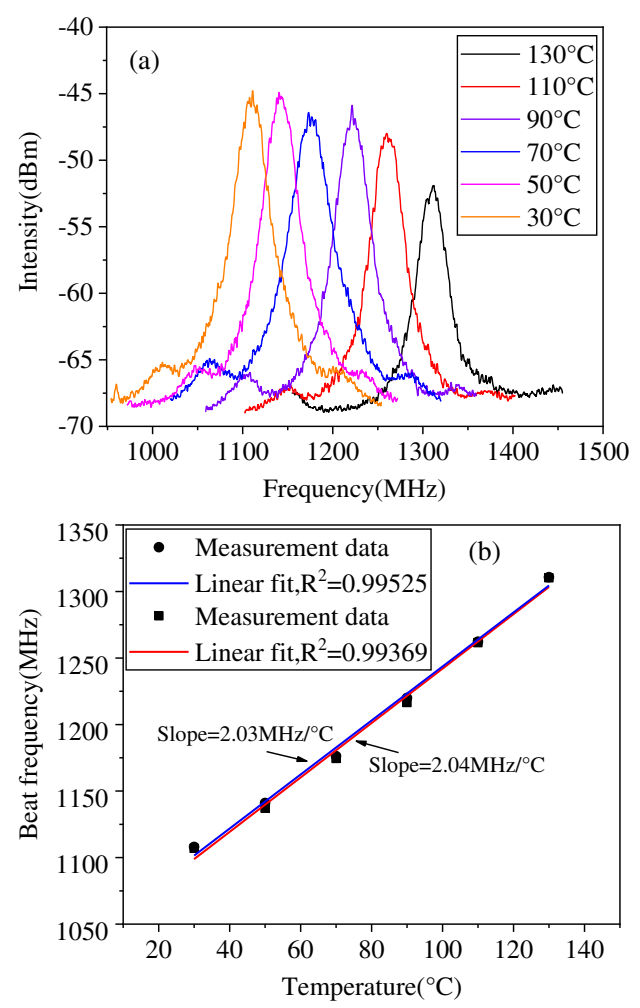

Fig. 9. (a) Shift of BFS with center frequency of $1092 \mathrm{MHz}$; (b) comparison of the two fitting results.

\section{Conclusions}

In summary, an MI compound cavity fiber laser sensor with RF detection has been proposed and demonstrated. In the system, the BFS is generated by MLM in the laser cavity. Heating SMF\#1 leads to a decrease in the arm length difference $\Delta L$ of $M I$ and an 
increase in the BFS envelope spacing $\Delta f$. By measuring the shift of the BFS envelope, the change in temperature can be readout. The experiment results show that the sensitivity of the system can reach $2.04 \mathrm{MHz} /{ }^{\circ} \mathrm{C}$ at $1106 \mathrm{MHz}$. This system uses a fiber optical MI to form a laser composite cavity structure, which greatly improves the sensitivity of fiber-optic sensing. RF detection is used to demodulate the MI sensor system. Compared with traditional optical domain detection, this method does not require expensive optical components and complex demodulation techniques, which reduces the cost of the system and improves the stability.

\section{Acknowledgement}

This work was supported by the National Natural Science Foundation of China (NSFC) (No. 62075057) and the Program for Innovative Research Team (in Science and Technology) in University of Henan Province (No. 21IRTSTHN011).

\section{References}

1. H. Sun, J. Zhang, Q. Rong, D. Feng, Y. Du, X. Zhang, D. Su, L. Zhou, Z. Feng, $\mathrm{X}$. Qiao, and M. Hu, "A hybrid fiber interferometer for simultaneous refractive index and temperature measurements based on Fabry-Perot/Michelson interference," IEEE Sens. J. 13, 2039 (2013).

2. J. Yuan, K. Zhang, W. Yao, B. Liu, Q. Li, Z. Zhang, K. Zhang, R. Chen, and S. Wang, "A simple fiber lateral stress sensor based on polarization-maintaining fiber embedded Michelson interferometer assisted by silicon rubber sheets," Optik 203, 164008 (2019).

3. D. Feng, M. Liu, W. Feng, and B. Li, "Michelson liquid-level sensor based on cascaded no-core fiber and single-mode fiber structure," Optik 206, 163746 (2019).

4. F. Xie, J. Ren, Z. Chen, and Q. Feng, "Vibration-displacement measurements with a highly stabilised optical fiber Michelson interferometer system," Opt. Laser Technol. 42, 208 (2010).

5. O. Frazão, S. F. Silva, J. Viegas, J. M. Baptista, J. L. Santos, and P. Royet, “A hybrid Fabry-Perot/Michelson interferometer sensor using a dual asymmetric core microstructured fiber," Meas. Sci. Technol 21, 025205 (2010).
6. M. Shao, H. Sun, J. Liang, L. Han, and D. Feng, "In-fiber Michelson interferometer in photonic crystal fiber for humidity measurement," IEEE Sens. J. 21, 1561 (2021).

7. H. Meng, W. Shen, G. Zhang, X. Wu, W. Wang, C. Tan, and X. Huang, "Michelson interferometer-based fiber-optic sensing of liquid refractive index," Sens. Actuators B Chem. 160, 720 (2011).

8. S. Zhang, Y. Liu, H. Guo, A. Zhou, and L. Yuan, "Highly sensitive vector curvature sensor based on two juxtaposed fiber Michelson interferometers with Vernier-like effect," IEEE Sens. J. 19, 2148 (2019).

9. X. Wang, T. Chen, D. Meng, and F. Wang, "A simple FBG Fabry-Perot sensor system with high sensitivity based on fiber laser beat frequency and Vernier effect," IEEE Sens. J. 21, 71 (2021).

10. J. T. Kringlebotn, W. H. Loh, and R. I. Laming, "Polarimetric $\mathrm{Er}^{3+}$-doped fiber distributed-feedback laser sensor for differential pressure and force measurements," Opt. Lett. 21, 1869 (1996).

11. H. K. Kim, S. K. Kim, H. G. Park, and B. Y. Kim, "Polarimetric fiber laser sensors," Opt. Lett. 18, 317 (1993).

12. B. Guan, L. Jin, Y. Zhang, and H. Y. Tam, "Polarimetric heterodyning fiber grating laser sensors," J. Light. Technol. 30, 1097 (2012).

13. S. Liu, Z. Yin, L. Zhang, L. Gao, X. Chen, and J. Cheng, "Multilongitudinal mode fiber laser for strain measurement," Opt. Lett. 35, 835 (2010).

14. W. Huang, S. Feng, W. Zhang, and F. Li, "DFB fiber laser static strain sensor based on beat frequency interrogation with a reference fiber laser locked to a FBG resonator," Opt. Express 24, 12321 (2016).

15. S. Miao, W. Zhang, W. Huang, and Y. Song, "High-resolution static strain sensor based on random fiber laser and beat frequency interrogation," IEEE Photonics Technol. Lett. 31, 1530 (2019).

16. H. Luo, D. Liu, T. Liu, D. Lyu, and Q. Sun, "Beat frequency characterization and stabilization for short-cavity DBR fiber grating laser based sensor," IEEE Photonics J. 9, 2725309 (2017).

17. J. Tian, M. Hou, Y. Jiang, H. Luo, and C. Tang, "Fiber ring laser cavity for strain sensing via beat frequency demodulation," Opt. Commun. 476, 126326 (2020).

18. J. Zhang, G. D. Peng, L. Yuan, and W. Sun, "Composite-cavity-based FabryPerot interferometric strain sensors," Opt. Lett. 32, 1833 (2007).

19. J. Zhang, Q. Chai, Q. Hao, Y. Ge, X. Li, Q. Li, W. Sun, L. Yuan, and G. Peng, "Composite cavity fiber laser sensors based on weak feedback," Appl. Opt. 50, 5059 (2011).

20. Y. Gao, S. Liu, Y. Ni, H. Wu, and X. Chen, "Multi-longitudinal mode fiber laser sensor system with high signal-to-noise ratio based on laser modes control," Opt. Express 27, 11776 (2019).

21. Z. Yin, L. Gao, S. Liu, L. Zhang, F. Wu, L. Chen, and X. Chen, "Fiber ring laser sensor for temperature measurement," J. Light. Technol. 28, 3403 (2011). 